\title{
Topological sensitivity of the objective function defined on morphing boundaries of two-dimensional heat conduction problems
}

\author{
G. Jing ${ }^{1}$, H. Isakari ${ }^{1}$, T. Matsumoto ${ }^{1}$, T. Yamada $^{2} \&$ T. Takahashi ${ }^{1}$ \\ ${ }^{I}$ Nagoya University, Japan \\ ${ }^{2}$ Kyoto University, Japan
}

\begin{abstract}
A topological sensitivity expression is presented for the objective function defined as a boundary integral of temperature and heat flux on the morphing boundaries which are newly generated during the topology optimization process. Since the objective function is defined by using only the boundary quantities, the boundary element method (BEM) is used for the heat conduction analyses of twodimensional media. A level set-based topology optimization method is assumed to obtain the optimum shape in two-dimensional heat conduction problems. The shape of the domain being morphed during the updating process of optimization can be extracted from the iso-surface of the level set function. The distribution of the level set function is obtained by solving the evolution equation of the level set function defined over a fixed design domain. The derivative of the level set function with respect to fictitious time is assumed to be proportional to the topological sensitivity of the objective function. In this paper, the objective function is considered to be defined on the newly generated morphing boundaries in the optimization process. The topological sensitivity for this case is derived and is verified through a numerical demonstration.

Keywords: level set method, topology optimization, heat conduction problems, topological sensitivity, boundary element method.
\end{abstract}

\section{Introduction}

Topology optimization has widely been studied in recent years and has shown a significant ability in structural optimization [1] since it allows not only the 
boundary shapes but also the topology changes of the design domain. For controlling the shape and topology of the material, level set method is successfully used. It enables to have a clear boundary expression and can effectively avoid the gray scale problems caused by using the approaches based on density distributions. Later, Yamada et al. [2] proposed a topology optimization method using a level set model incorporating a fictitious interface energy derived from phase field concept, and by adding a regularization term to the objective function, controlling the geometric complexity of the expected optimal configurations of the structure was enabled.

The finite element method (FEM) has been used as a solver of the physical simulation in most of the foregoing topology optimizations [3-6]. Because the meshing cost is rather high in FEM, the entire fixed design domain has been kept used as the computational domain by giving sufficiently small values of the material properties to the finite elements where the material does not exist any more after some iterative process of topology optimization. But this trick does not work well in some applications like harmonic vibrations in which a small difference in the distribution of the material property dramatically affects frequency responses. To have a better result, we have to regenerate FEM mesh at every iterative step of optimization and give the corresponding boundary condition to the newly generated morphing boundaries.

The boundary element method (BEM) [7] may be a strong alternative to FEM when the objective function is defined only on the boundary of the structure because its meshing cost is dramatically lower than that in FEM, particularly for the mesh regeneration for morphing boundaries. In fact, BEM has been used in a topology optimization for potential problems [8], and applied recently to heat problems [9].

In the previous works, we have successfully applied the method to heat conduction problems to have topology changes of the heat transfer boundaries. In this paper, we extend the previous method so that the objective function can be defined even on the newly generated (morphing) boundaries. The topological sensitivity corresponding to the new objective function is derived accordingly and is verified through a numerical demonstration.

\section{Formulations}

\subsection{Topology optimization}

The level set method is an approach to represent material shapes by using level set function that is a scalar function of point, $\phi(x)$, defined as follows:

$$
\begin{cases}0<\phi(x) \leq 1, & x \in \Omega \backslash \Gamma, \\ \phi(x)=0, & x \in \Gamma, \\ -1 \leq \phi(x)<0, & x \in D \backslash \Omega,\end{cases}
$$


where $D, \Omega$ and $\Gamma$ denote the fixed design domain, the material domain and the boundary of $\Omega$, respectively, as shown in Figure 1 .

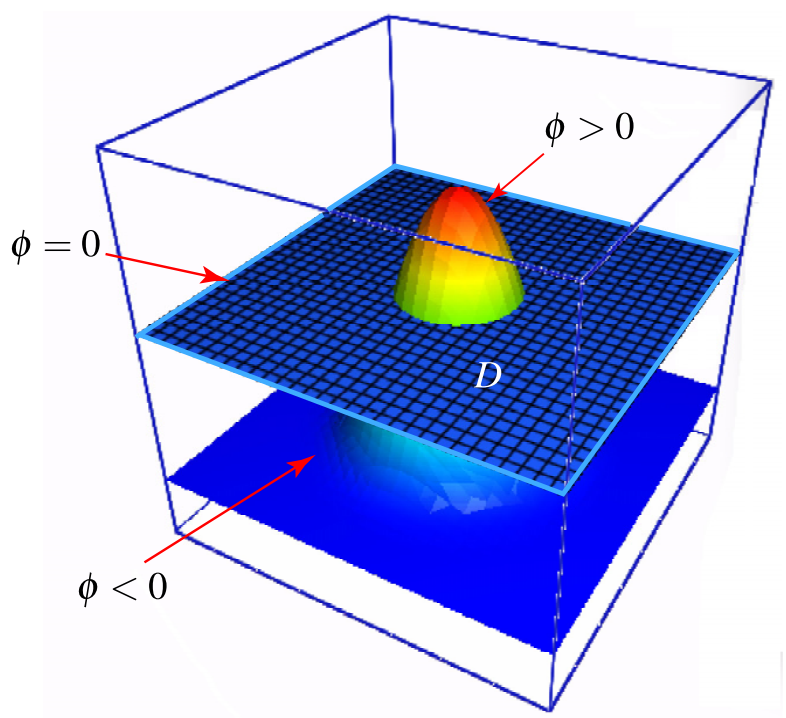

Figure 1: Level set function defined the fixed design domain $D$.

We consider the following optimization problem where the objective functional is defined on the boundary $\Gamma$.

$$
\inf _{\phi} I=\int_{\Gamma} f(u, q) d \Gamma
$$

subject to

$$
\begin{aligned}
& \text { D.E.: } \quad \nabla \cdot(-k \nabla u)=0 \quad \text { in } \Omega \text {, } \\
& \text { B.C.: } u=\bar{u} \text { on } \Gamma_{u} \text {, } \\
& q=-k \frac{\partial u}{\partial n}=\bar{q} \quad \text { on } \Gamma_{q}, \\
& q=h\left(u-u_{\infty}\right) \quad \text { on } \Gamma_{h} .
\end{aligned}
$$

and

$$
G=\int_{D} H(\phi(x)) d \Omega-G_{\max } \leq 0
$$

where $f(u, q)$ is a function of $u$ and $q$ defined on $\Gamma$ or part of $\Gamma . u$ and $q=-k \frac{\partial u}{\partial n}$ are the temperature and heat flux, respectively, $n$ is the outward normal direction to $\Gamma, u_{\infty}$ is the ambient temperature, $k$ is the thermal conductivity, $h$ is the heat 
transfer coefficient, and $H$ is the Heaviside function. $G_{\max }$ is the admissible upper limit of the area of the material region $\Omega$.

The objective functional Eq. (2) can be augmented by considering Eq. (3) together, as follows:

$$
\inf _{\phi} J=I(\phi)+\int_{D} \mu \nabla \cdot(-k \nabla u) d \Omega,
$$

where $\mu$ is the adjoint variable working as Lagrange's multiplier.

To obtain the optimum distribution of the level set function $\phi$ minimizing $J$, we assume that the distribution of $\phi$ changes in accordance with a sort of time-like variable $t$ corresponding to the morphing of the material domain and that $\partial \phi / \partial t$ is proportional to $\mathcal{T}$, the topological sensitivity of $J$. $\partial \phi / \partial t$ is also assumed to be proportional to $\lambda$ that is a penalty parameter for $0 \leq \lambda \leq 1$ and $\nabla^{2} \phi$ that is considered as the curvature of $\phi$. When the topological sensitivity value is negative, removing the infinitely small cavity at that point results in decreasing the objective function value. Hence, $\partial \phi / \partial t$ has the same sign as the topological sensitivity. Also, by giving the same sign of $\nabla^{2} \phi$ to $\partial \phi / \partial t$, the variation of $\phi$ becomes smoother. However, since the positive value of the penalty parameter $\gamma$ implies that the volume constraint is broken, $\partial \phi / \partial t$ should be proportional to the negative sign of $\gamma$. Thus, we have the equation for time evolution of the level set function $\phi$, as follows:

$$
\frac{\partial \phi}{\partial t}=K\left(\mathcal{T}-\lambda+\tau \nabla^{2} \phi\right) \quad \text { in } D,
$$

where $\tau$ is a regularization parameter that can control the curvature distribution of $\phi$.

The boundary condition for Eq. (9) can be set as follows:

$$
\begin{gathered}
\frac{\partial \phi}{\partial n}=0 \quad \text { on } \partial D \backslash \Gamma_{N}, \\
\phi=1 \quad \text { on } \Gamma_{N},
\end{gathered}
$$

where $K>0$ is a constant, $\Gamma_{N}$ represents non design boundaries of the fixed design domain $D$.

\subsection{Topological derivative}

The augmented objective function $J$ can be rewritten, after integrating by parts, as follows:

$$
J=\int_{\Gamma} f d \Gamma+\int_{\Gamma} \mu q d \Gamma-\int_{\Omega} \nabla \mu \cdot(-k \nabla u) d \Omega .
$$

The objective function suffers from a change, denoted by $\delta J$, when an infinitesimal region $\Omega_{\epsilon}$ is removed from $\Omega$. After some manipulations, we obtain 
as

$$
\begin{aligned}
\delta J= & \int_{\Gamma_{u}}\left(\mu+\frac{\partial f}{\partial q}\right) \delta q d \Gamma-\int_{\Gamma_{q}}\left(\eta-\frac{\partial f}{\partial u}\right) \delta u d \Gamma \\
& -\int_{\Gamma_{h}}\left[\eta-h\left(\mu+\frac{1}{h} \frac{\partial f}{\partial u}+\frac{\partial f}{\partial q}\right)\right] \delta u d \Gamma \\
& +\int_{\Gamma_{\epsilon}}\left(f+\frac{\partial f}{\partial u} \delta u+\frac{\partial f}{\partial q} \delta q\right) d \Gamma-\int_{\Omega \backslash \Omega_{\epsilon}}[\nabla \cdot(-k \nabla \mu)] \delta u d \Gamma \\
& -\int_{\Gamma_{\epsilon}} \eta \delta u d \Gamma+\int_{\Gamma_{\epsilon}} \mu(q+\delta q) d \Gamma+\int_{\Omega_{\epsilon}} \nabla \mu \cdot(-k \nabla u) d \Omega
\end{aligned}
$$

where

$$
\eta=-k \frac{\partial \mu}{\partial n}=-k \nabla \mu \cdot \mathbf{n}
$$

We assume that the adjoint variable $\mu$ is the solution of the following boundary value problem:

$$
\begin{array}{ll}
\nabla \cdot(-k \nabla \mu)=0 & \text { in } \Omega, \\
\mu=-\frac{\partial f}{\partial q} & \text { on } \Gamma_{u} \\
\eta=\frac{\partial f}{\partial u} & \text { on } \Gamma_{q}, \\
\eta=h\left(\mu+\frac{1}{h} \frac{\partial f}{\partial u}+\frac{\partial f}{\partial q}\right) & \text { on } \Gamma_{h} .
\end{array}
$$

Also, after some lengthy manipulations, we obtain the leading terms of $\delta u$ and $\delta q$ on $\Gamma_{\epsilon}$ as follows:

$$
\begin{gathered}
\delta u=\epsilon \frac{h\left(u^{0}-u_{\infty}\right)}{k} \ln r+\frac{\epsilon^{2}}{r} u_{, 1}^{0} \cos \theta+\frac{\epsilon^{2}}{r} u_{, 2}^{0} \sin \theta \\
\delta q=\frac{\epsilon}{r} h\left(u^{0}-u_{\infty}\right)-\frac{\epsilon^{2} k}{r^{2}} u_{, 1}^{0} \cos \theta-\frac{\epsilon^{2} k}{r^{2}} u_{, 2}^{0} \sin \theta
\end{gathered}
$$

Using these formulas, we obtain

$$
\begin{aligned}
\delta J= & 2 \pi \epsilon \mu^{0} h\left(u^{0}-u_{\infty}\right) \\
& +\epsilon \int_{0}^{2 \pi} f d \theta+\epsilon \int_{0}^{2 \pi} \frac{\partial f}{\partial u}\left(u_{, 1}^{0} \cos \theta+u_{, 2}^{0} \sin \theta\right) d \theta \\
& +\int_{0}^{2 \pi} \frac{\partial f}{\partial q}\left[h\left(u^{0}-u_{\infty}\right)-k\left(u_{, 1}^{0} \cos \theta+u_{, 2}^{0} \sin \theta\right)\right] d \theta
\end{aligned}
$$


For a special case when $f$ is given as

$$
f=(u-\hat{u})^{2},
$$

where $\hat{u}$ is the target temperature on the newly generated boundary $\Gamma_{\epsilon}, \delta J$ results in

$$
\delta J=2 \pi \epsilon \mu^{0} h\left(u^{0}-u_{\infty}\right)+2 \pi \epsilon(u-\hat{u})^{2}+o\left(\epsilon^{2}\right),
$$

from which we obtain the corresponding topological sensitivity as follows:

$$
\mathcal{T}=\lim _{\epsilon \rightarrow 0} \frac{\delta J}{2 \pi \epsilon}=\mu^{0} h\left(u^{0}-u_{\infty}\right)+\left(u^{0}-\hat{u}\right)^{2} .
$$

\section{Numerical simulation}

The correctness of the topological sensitivity expression given by Eq. (24) is verified.

Let us consider a design domain initially filled with the material entirely in the area of $4.0[\mathrm{~m}] \times 4.0[\mathrm{~m}]$ as shown in Figure 2 . Temperature boundary condition is given for the left and top edges of the design domain with a prescribed temperature $\bar{u}=50^{\circ} \mathrm{C}$, while heat flux boundary condition is given for the bottom and right edges with a prescribed heat flux $\bar{q}=50\left[\mathrm{~W} / \mathrm{m}^{2}\right]$. The thermal conductivity of the domain is assumed as $k=1.0[\mathrm{~W} /(\mathrm{m} \cdot \mathrm{K})]$. We compare the topological derivative values calculated by Eq. (24) with the approximate values calculated by finite difference of the values of the objective functions both for the original domain and the domain from which a small circular hole is actually deleted. A heat transfer boundary condition with the ambient temperature $u_{\infty}=45^{\circ} \mathrm{C}$ and the heat transfer coefficient $h=0.001\left[\mathrm{~W} /\left(\mathrm{m}^{2} \cdot \mathrm{K}\right)\right]$ is considered on the circular hole.

The objective function is defined as

$$
I=\int_{\Gamma_{h}}(u-\hat{u})^{2} d \Gamma,
$$

where $\hat{u}=15^{\circ} \mathrm{C}$ is the target temperature.

The boundary of the square domain is discretized uniformly with $40 \times 4=160$ quadratic conforming elements. Also, $40 \times 40=1600$ grids are generated in the fixed design domain. The topological derivative values are calculated at the sample internal grid points shown in Figure 3.

The approximate values of the topological derivative is calculated by the following formula:

$$
J_{\text {approx }}^{\prime}=\frac{J_{\text {original }}-J_{\text {hole }}}{2 \pi \epsilon^{*}},
$$

where $F_{\text {original }}$ and $F_{\text {hole }}$ denote the values of the objective function before and after the hole is created. $\epsilon^{*}=0.0001[\mathrm{~m}]$ is the radius of the hole, and the boundary of the hole is divided into 32 quadratic elements when $J_{\text {hole }}$ is calculated using the boundary element method. As shown in Figure 4, the topological derivative 


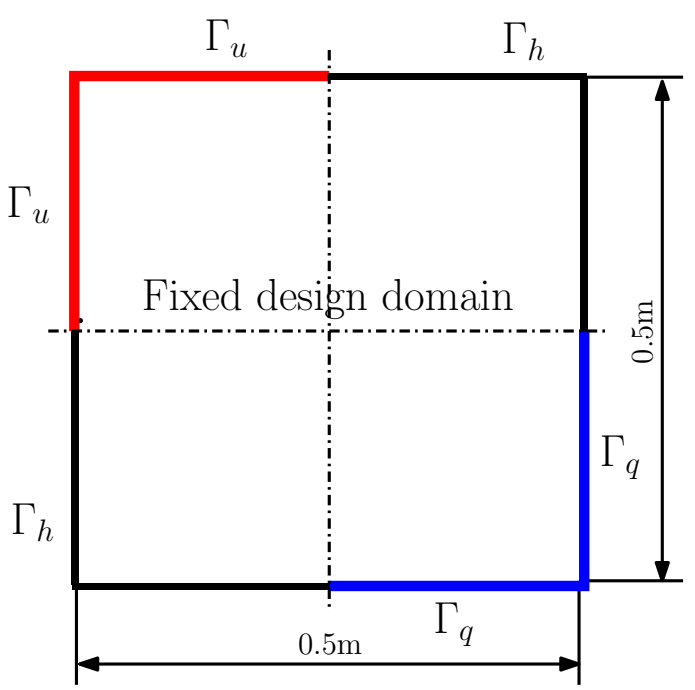

Figure 2: Fixed design domain used to verify the topological sensitivity expression.

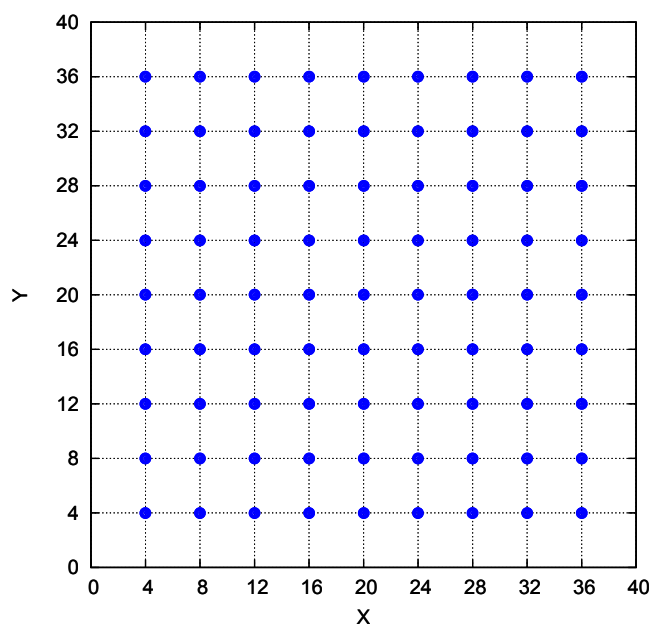

Figure 3: Sample points for verifying the topological sensitivity expression.

values obtained using the proposed approach are in very good agreement with those obtained with the finite difference formula, of which the maximum error is less than $1 \%$, thus the present formula of the topological derivative is verified. 


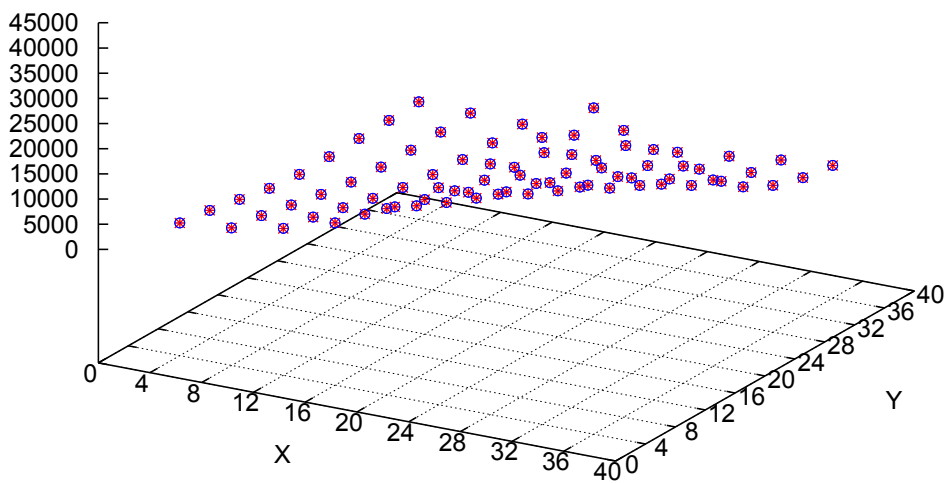

Figure 4: Comparison of the topological sensitivities obtained by its present explicit expression with those obtained by the finite difference scheme.

\section{Concluding remarks}

In this work, the topological sensitivity expression for heat conduction problems with objective function defined also on the newly generated morphing boundaries, on which heat transfer boundary condition is prescribed, is derived and verified. The correctness of the derived expression for the topological sensitivity is demonstrated by calculating those values at the internal points in comparison with those obtained by finite difference scheme.

\section{References}

[1] M.P. Bendsøe, O. Sigmund, "Topology optimization-Theory, Methods and Applications", Springer, Berlin-Heidelberg, 2003.

[2] T. Yamada, K. Izui, S. Nishiwaki, A. Takezawa, "A topology optimization method based on the level set method incorporating a fictitious interface energy", Computer Methods in Applied Mechanics and Engineering, 199, 2876-2891, 2010.

[3] X. Xing, Y.W. Michael, "Structural topology optimization using finite element based level set method", Proceeding of NAFEMS World Congress, 2009. 
[4] K. Sebastian, M. Kurt, "Levelset based fluid topology optimization using the extended finite element method", Structural and Multidisciplinary Optimization, 46, 311-326, 2012.

[5] T. Yamada, K. Izui, S. Nishiwaki, A. Takezawa, "A topology optimization method based on the level set method incorporating a fictitious interface energy", Computer Methods in Applied Mechanics and Engineering, 199, 2876-2891, 2010.

[6] G.H. Yoon, J.S. Jensen, O. Sigmund, "Topology optimization of acousticstructure interaction problems using a mixed finite element formulation", International journal for numerical methods in engineering, 70, 1049-1075, 2007.

[7] W.S. Hall, "Boundary Element Method", Springer, 1994.

[8] T. Matsumoto, T. Yamada, S. Shichi, T. Takahashi, "A study on topology optimization using the level-set function and BEM", Boundary Elements and Other Mesh Reduction Methods XXXIV, C.A. Brebbia and D. Poljak (Eds.), 123-133, 2012.

[9] G. Jing, T. Matsumoto, T. Takahashi, H. Isakari, T. Yamada, “Topology optimization for 2D heat conduction problems using boundary element method and level set method", Transactions of JASCOME, 13, 91-96, 2013.

[10] T. Yamada, K. Izui, S. Nishiwaki, "A Level Set-Based Topology Optimization Method for Maximizing Thermal Diffusivity in Problems Including Design-Dependent Effects”, Journal of Mechanical Design, 133, 031011, 2011.

[11] A. N. Tikhonov, V.Y. Arsenin, "Solutions of Ill-Posed Problems", Winston and Sons, Washington, D.C., 1997. 